\title{
First-dose mRNA vaccination is sufficient to reactivate immunological memory to SARS-CoV-2 in subjects who have recovered from COVID-19
}

\author{
Alessio Mazzoni, ${ }^{1}$ Nicoletta Di Lauria, ${ }^{2}$ Laura Maggi, ${ }^{1}$ Lorenzo Salvati, ${ }^{1}$ Anna Vanni, ${ }^{1}$ Manuela Capone, ${ }^{1}$ Giulia Lamacchia, ${ }^{1}$ \\ Elisabetta Mantengoli, ${ }^{2}$ Michele Spinicci, ${ }^{1,2}$ Lorenzo Zammarchi, ${ }^{1,2}$ Seble Tekle Kiros, ${ }^{1,2}$ Arianna Rocca, ${ }^{1}$ Filippo Lagi, ${ }^{1}$ \\ Maria Grazia Colao, ${ }^{3}$ Paola Parronchi, ${ }^{1,4}$ Cristina Scaletti, ${ }^{1}$ Lucia Turco, ${ }^{5}$ Francesco Liotta, ${ }^{1,4}$ Gian Maria Rossolini, ${ }^{1,3}$ \\ Lorenzo Cosmi, ${ }^{1,4}$ Alessandro Bartoloni, ${ }^{1,2}$ and Francesco Annunziato ${ }^{1,6}$ for the COVID-19 Research Group \\ Department of Experimental and Clinical Medicine, University of Florence, Florence, Italy. ${ }^{2}$ Infectious and Tropical Diseases Unit, ${ }^{3}$ Microbiology and Virology Unit, ${ }^{4}$ Immunology and Cell Therapy Unit, \\ ${ }^{5}$ Health Director, and ${ }^{6}$ Flow Cytometry Diagnostic Center and Immunotherapy, Careggi University Hospital, Florence, Italy.
}

The characterization of the adaptive immune response to COVID-19 vaccination in individuals who recovered from SARSCoV-2 infection may define current and future clinical practice. To determine the effect of the 2-dose BNT162b2 mRNA COVID-19 vaccination schedule in individuals who recovered from COVID-19 (COVID-19-recovered subjects) compared with naive subjects, we evaluated SARS-CoV-2 Spike-specific T and B cell responses, as well as specific IgA, IgG, IgM, and neutralizing antibodies titers in 22 individuals who received the BNT162b2 mRNA COVID-19 vaccine, 11 of whom had a previous history of SARS-CoV-2 infection. Evaluations were performed before vaccination and then weekly until 7 days after second injection. Data obtained clearly showed that one vaccine dose is sufficient to increase both cellular and humoral immune response in COVID-19-recovered subjects without any additional improvement after the second dose. On the contrary, the second dose proved mandatory in naive subjects to further enhance the immune response. These findings were further confirmed at the serological level in a larger cohort of naive $(n=68)$ and COVID-19-recovered $(n=29)$ subjects, tested up to $\mathbf{5 0}$ days after vaccination. These results question whether a second vaccine injection in COVID-19-recovered subjects is required, and indicate that millions of vaccine doses may be redirected to naive individuals, thus shortening the time to reach herd immunity.

\section{Introduction}

As of April 4, 2021, more than 130.4 million people have been diagnosed with COVID-19 worldwide, with more than 2.8 million confirmed deaths (1). COVID-19 is associated with high transmission and rising numbers of cases of acute respiratory distress are threatening to overwhelm global health care capacity (2-5). Up to now, few COVID-19 vaccines based on mRNA or adenovirus have been approved by international and national medicines agencies. For this reason, we are currently facing some difficulties in producing a number of vaccine doses adequate to meet current needs (6-9). Approved COVID-19 mRNA vaccines require a first dose followed 3 to 4 weeks later by a second injection $(6,7)$. In many countries, vaccinations are carried out on all volunteer subjects regardless of past medical history of testing positive for SARS-

\section{Related Commentary: https://doi.org/10.1172/JCI150135}

Authorship note: AM and NDL contributed equally to this work. $\mathrm{AB}$ and FA are cosenior authors.

Conflict of interest: The authors have declared that no conflict of interest exists. Copyright: @ 2021, American Society for Clinical Investigation.

Submitted: March 1, 2021; Accepted: April 26, 2021; Published: June 15, 2021.

Reference information: J Clin Invest. 2021;131(12):e149150.

https://doi.org/10.1172/JCl149150.
CoV-2 infection (10). This approach raises 2 main questions: is it necessary to vaccinate those who recovered from COVID-19, and if so, is it necessary to administer them a second dose of vaccine?

In this manuscript, we evaluated SARS-CoV-2 Spike-specific $\mathrm{T}$ and $\mathrm{B}$ cells responses, as well as IgG, IgA, IgM, and neutralizing antibodies specific titers in 22 individuals, 11 of whom had a previous history of COVID-19 (COVID-19-recovered subjects). All recruited subjects were evaluated before receiving the BNT162b2 mRNA COVID-19 vaccine and then weekly until 7 days after the second injection (administered 21 days after the first dose). Data clearly showed that one vaccine dose is sufficient to increase both cellular and humoral immune response in COVID-19-recovered subjects without any additional improvement after the second dose. These results question whether a second vaccine injection in COVID-19-recovered subjects is indeed required and indicate that millions of vaccine doses may be redirected to naive individuals.

\section{Results and Discussion}

During the first 2 months of the vaccination campaign against COVID-19 using the BNT162b2 mRNA vaccine in Florence, Italy, we recruited 22 healthcare workers, 11 of whom had a previous history of SARS-CoV-2 infection. The demographic and clinical characteristics of the recruited subjects are detailed in Supplemental Table 1; supplemental material available online with this 
A
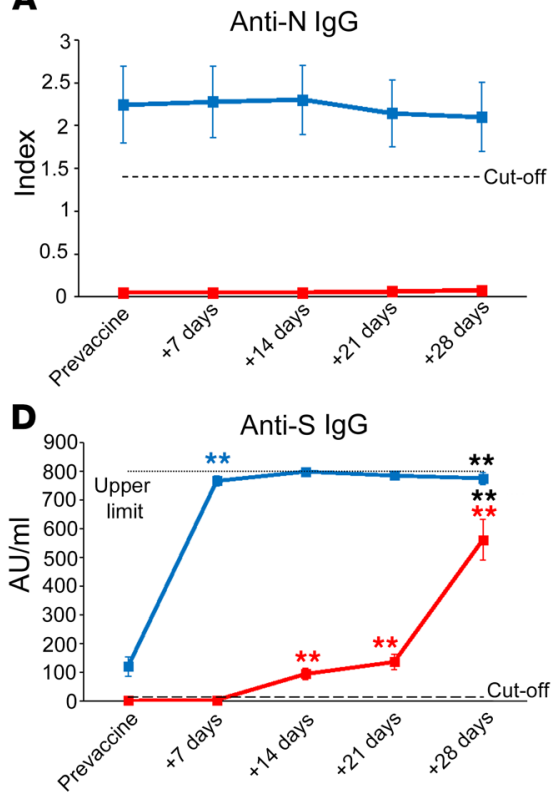

B

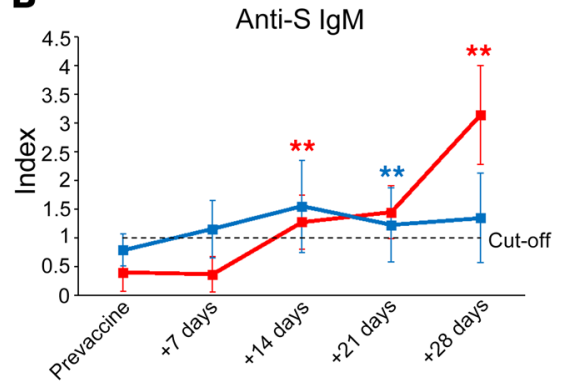

E

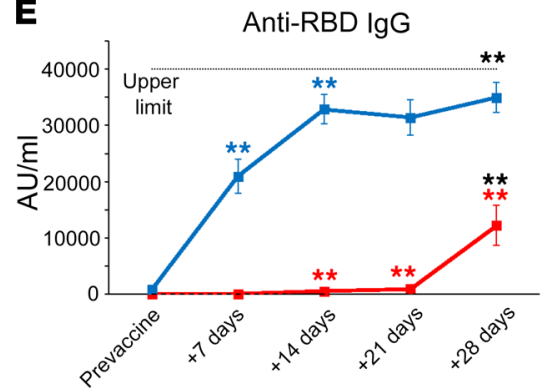

C Naive subjects = Recovered COVID-19 subjects

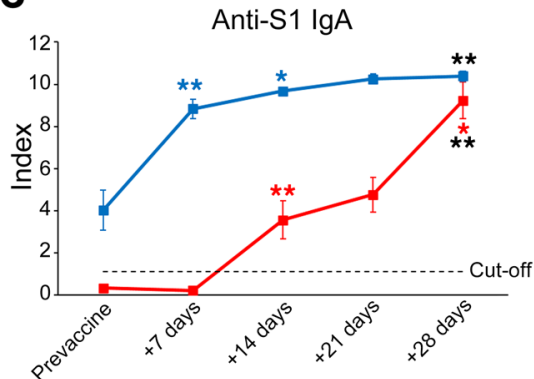

$\mathbf{F}$

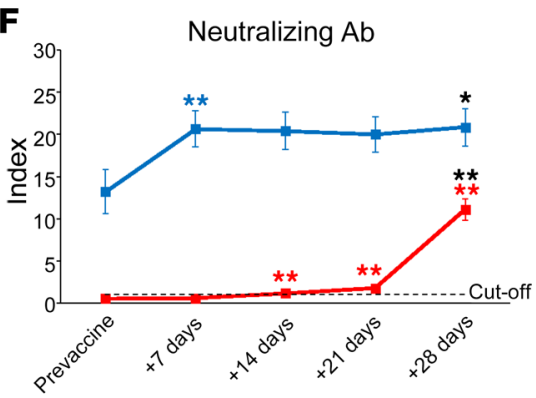

Figure 1. Evaluation of anti-SARS-CoV-2 serum antibody levels. Evaluation of anti-N IgG (A), anti-S IgM (B), anti-S1 IgA (C), anti-S IgC (D), anti-RBD IgG (E), and S-neutralizing antibodies (F) in naive (red lines) and COVID-19-recovered (blue lines) subjects before and 7, 14, 21, 28 days after first vaccine administration. Data are represented as mean \pm SEM from 11 naive and 11 COVID-19-recovered subjects. Dashed lines represent cut-off values, dotted lines are upper detection limits. Blue and red asterisks refer to paired statistics within each study group compared with the previous time point in the kinetic. Black asterisks at day 28 represent paired statistic compared with prevaccine point. ${ }^{*} P<0.05$; ${ }^{*} P<0.01$ calculated with Wilcoxon's signed rank test.

article; https://doi.org/10.1172/JCI149150DS1. We collected a peripheral blood draw at basal time (time 0 , before injection of the first $30 \mu \mathrm{g}$ dose), then 7 days, 14 days, 21 days (before injection of the second $30 \mu \mathrm{g}$ dose), and 28 days later (Supplemental Figure 1). The 22 recruited subjects were evaluated at each time point for SARS-CoV-2-specific humoral response and the presence of Spike-specific $\mathrm{T}$ and $\mathrm{B}$ cells.

As shown in Figure 1A, antinucleoprotein (anti-N) IgG was present before vaccination only in COVID-19-recovered subjects, confirming their previous exposure to SARS-CoV-2 $(P<0.001$ with Mann-Whitney $U$ test). As expected, anti-N IgG levels remained stable over the study period in COVID-19-recovered subjects and undetectable in naive individuals (Figure 1A). On the other hand, anti-S IgM increased over time in naive subjects, while exhibiting no substantial changes in COVID-19-recovered individuals (Figure 1B). Prevaccination levels of anti-S IgA and IgG were detected only in COVID-19-recovered subjects $(P<0.01$ with Mann-Whitney $U$ test). In naive individuals, anti-S IgA and IgG increased progressively from 14 to 21 days following the first injection and grew again at day 28 , one week after the second dose. On the contrary, in COVID-19-recovered subjects, anti-S IgA and IgG massively increased at day 7 following first injection and remained stable over time (Figure 1, C and D). We also measured anti-RBD IgG, whose kinetic appeared similar to that of anti-S IgG in both naive and COVID-19-recovered individuals (Figure 1E). Interestingly, neutralizing antibodies were already present in the sera of COVID-19-recovered subjects before vaccination but significantly increased after the first BNT162b2 mRNA vaccine dose, and did not further grow after the second injection (Figure 1F). On the other side, naive subjects showed appreciable titers of neutralizing antibodies only 1 week after the administration of the second vaccine dose and did not reach the levels present in COVID-19-recovered subjects' sera (Figure $1 \mathrm{~F}$ ).

We evaluated by flow cytometry the frequency of B cells capable of recognizing SARS-CoV-2 Spike protein (Figure 2A and Supplemental Figure 2). As shown in Figure 2B, COVID-19-recovered subjects already presented significantly higher frequencies of circulating SARS-CoV-2 Spike-specific B cells before vaccination as compared with naive individuals $(P<0.001$ with Mann-Whitney U test). In COVID-19-recovered subjects, we observed a constant increase of the frequency of Spike-specific B cells up to 21 days, but then it significantly declined after the second injection (Figure $2 \mathrm{~B}$ ). On the contrary, naive individuals showed an appreciable increase of these cells only 1 week after the second dose (Figure 2B). Of note, the frequency of Spike-specific B cells at day 28 was significantly lower in naive than in COVID-19-recovered subjects $(P<0.001$ with Mann-Whitney $U$ test). As shown in Figure 2, C-E, Spike-specific B cells were predominantly CD $27^{+} \mathrm{IgG}^{+}$cells, but $\mathrm{CD} 27^{+} \mathrm{B}$ cells of the IgM and IgA isotype could also be identified. The kinetics of Spike-specific B cells expressing the 3 different immunoglobulin classes in COVID-19-recovered subjects was comparable to that described for total B cells (Figure 2, C-E). In naive subjects, the frequencies of Spike-specific $\mathrm{CD} 27^{+} \mathrm{IgG}^{+}$, $\mathrm{CD}_{27} 7^{+} \mathrm{IgM}^{+}$, and $\mathrm{CD} 27^{+} \operatorname{IgA}^{+}$cells increased only after the administration of the second vaccine dose (Figure 2, C-E).

These observations show that in COVID-19-recovered subjects the first injection of the BNT162b2 mRNA vaccine is sufficient to reactivate immunological memory. Moreover, naive indi- 

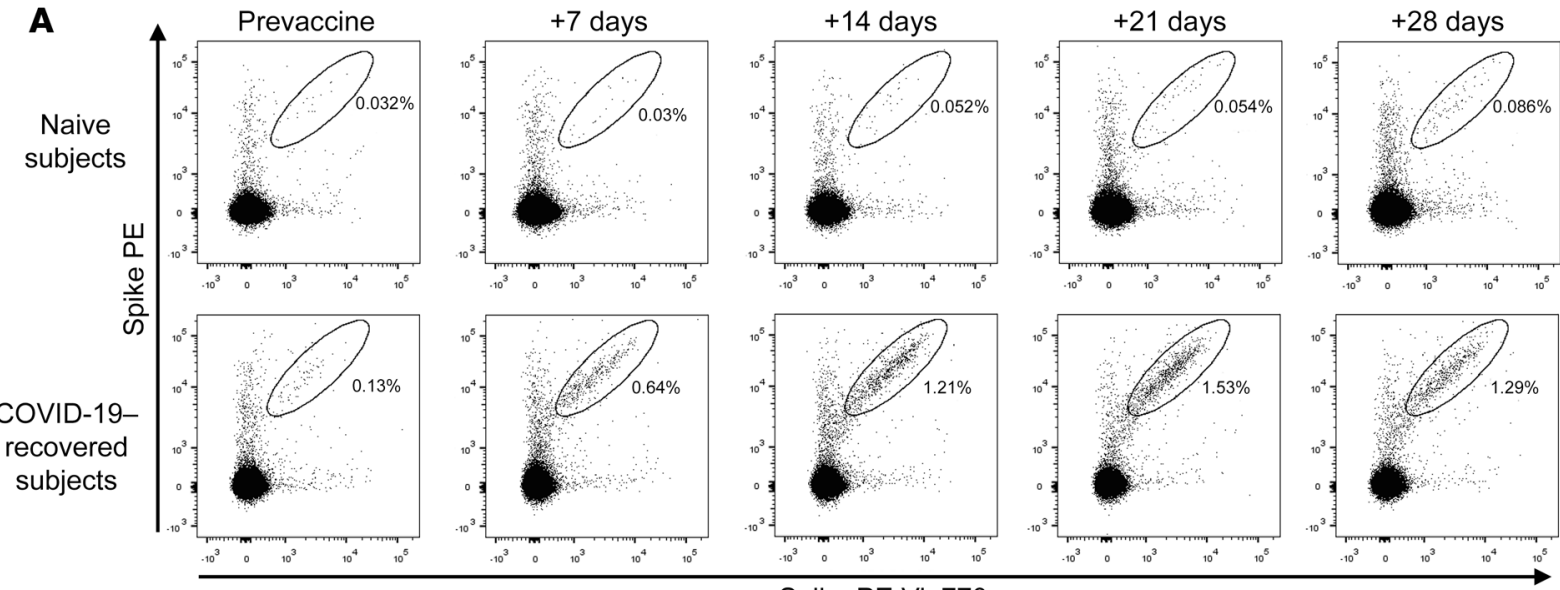

Spike PE Vio770
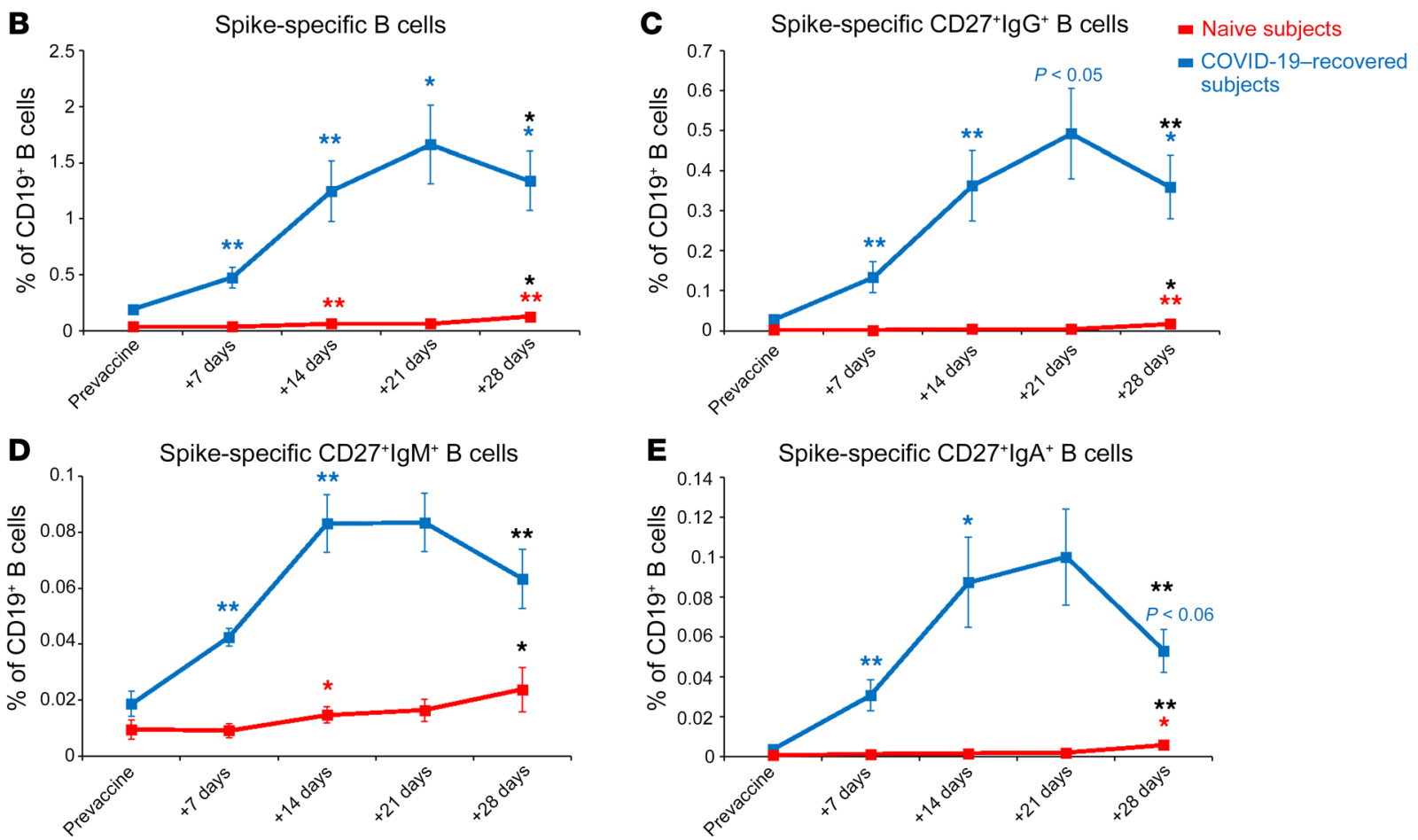

Figure 2. Detection of Spike-specific circulating B cells in naive and COVID-19-recovered vaccinated subjects. (A) Representative flow cytometric plots of Spike-specific B cells in 1 naive (upper row) and 1 COVID-19-recovered subject (lower row) before vaccination and 7, 14, 21, 28 days after the first injection. Kinetic analysis of frequencies of total (B), $C D 27^{+} \operatorname{IgG}{ }^{+}(\mathbf{C}), C D 27^{+} \operatorname{IgM}{ }^{+}(\mathbf{D})$, and $\mathrm{CD} 27^{+} \operatorname{IgA}{ }^{+}$(E) Spike-specific B cells in naive (red lines) and COVID-19-recovered (blue lines) subjects before and after vaccination. Data are mean \pm SE from 11 naive and 11 COVID-19-recovered subjects. Blue and red asterisks refer to paired statistics within each study group compared with the previous time point in the kinetic. Black asterisks at day 28 represent paired statistic compared with prevaccine point. ${ }^{*} P<0.05 ;{ }^{* *} P<0.01$ calculated with Wilcoxon's signed rank test.

viduals did not exhibit frequency of B cells and serum Ig levels comparable to those presented by COVID-19-recovered subjects, at least at day 28 after the first injection. This was especially true for antibodies with neutralizing capability.

We also studied the Spike-specific $\mathrm{CD} 4^{+} \mathrm{T}$ cell response, monitoring CD154 surface expression and the production of IL-2, IFN- $\gamma$, and TNF- $\alpha$ upon in vitro stimulation with peptide pools (Figure 3A and Supplemental Figure 3). Similarly to Spike-specific $\mathrm{B}$ cells, circulating Spike-specific $\mathrm{CD} 4^{+} \mathrm{T}$ cells were present in COVID-19-recovered subjects before vaccination, while they were absent in naive individuals $(P<0.01$ with Mann-Whitney $U$ test; Figure 3B). Moreover, in COVID-19-recovered subjects, we observed a significant increase already at day 7 after the first BNT162b2 mRNA vaccine administration, in contrast to naive individuals (Figure 3B). This observation was evident not only by evaluating the frequency of $\mathrm{CD} 4^{+} \mathrm{T}$ cells expressing $\mathrm{CD} 154$ and at least 1 of the 3 monitored cytokines (IL-2, IFN- $\gamma$, and TNF- $\alpha$; Figure $3 \mathrm{~B}$ ), but also by evaluating the frequency of $\mathrm{T} \mathrm{CD}^{+}$cells expressing CD154 in association with each individual cytokine (Figure 3, C-E). Unlike our findings for Spike-specific B cell 
A
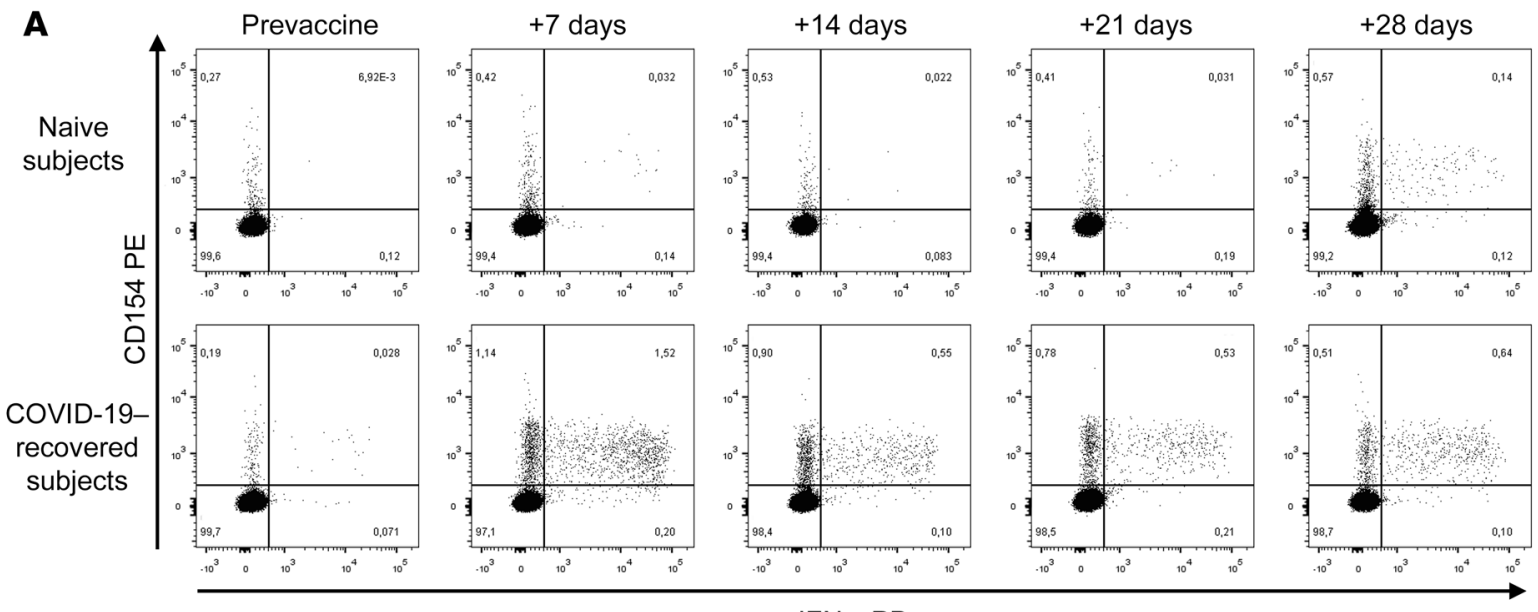

IFN- $\gamma \mathrm{PB}$

B

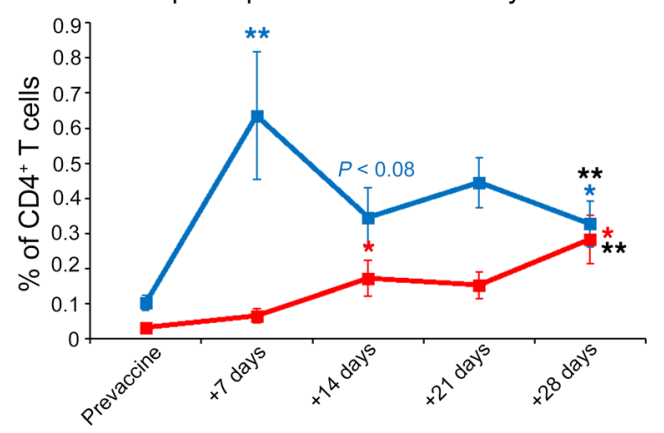

D

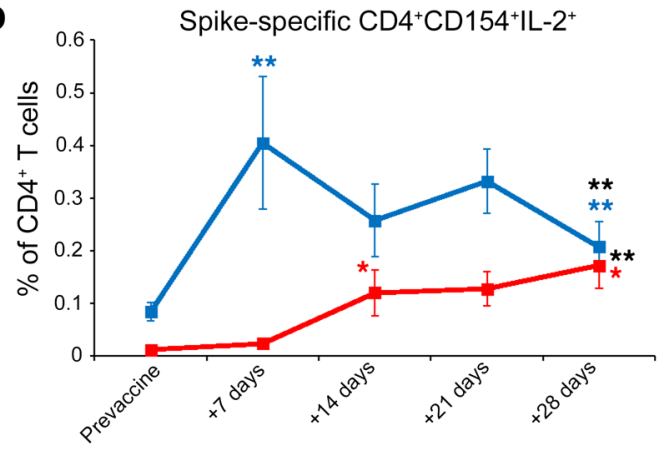
F $\begin{gathered}\text { Naive } \\ \text { subjects }\end{gathered}$

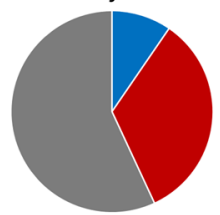

3 cytokines

2 cytokines

1 cytokine
COVID-19-

recovered subjects

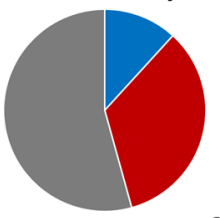

COVID-19-

Naive sujects recovered subjects $($ mean $\pm \mathrm{SE}) \quad($ mean $\pm \mathrm{SE})$

$9.6 \pm 1.0 \% \quad 11.7 \pm 2.2 \%$

$33.5 \pm 2.1 \% \quad 33.9 \pm 2.1 \%$

$56.9 \pm 2.5 \% \quad 54.4 \pm 4.1 \%$
C

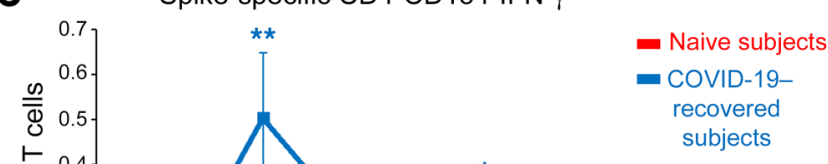

\section{E}

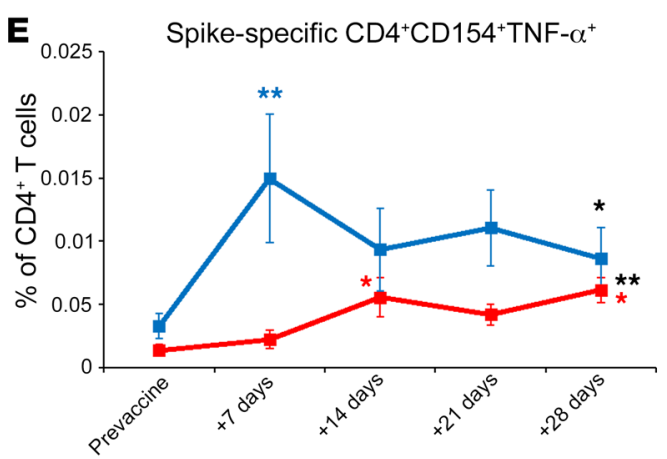

G

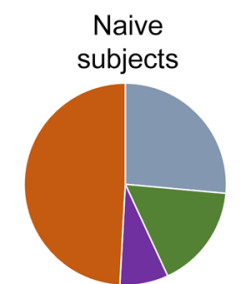

COVID-19-

recovered subjects

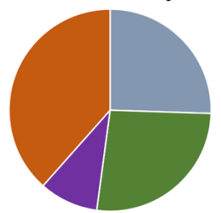

COVID-19-

Naive sujects recovered subjects

$($ mean \pm SE $) \quad($ mean \pm SE $)$

TIGIT-PD1- $49.1 \pm 2.8 \% \quad 38.4 \pm 8 \%$

TIGIT-PD1 ${ }^{+} \quad 26.4 \pm 3.9 \% \quad 25.6 \pm 5.3 \%$

$\mathrm{TIGIT}^{+} \mathrm{PD} 1^{+} \quad 16.7 \pm 2.5 \% \quad 26.6 \pm 4.9 \%$

TIGIT+PD1- $7.8 \pm 2.7 \% \quad 9.4 \pm 1.6 \%$ 
Figure 3. Detection of Spike-specific circulating CD4+ $\mathbf{T}$ cells in naive and COVID-19-recovered vaccinated subjects. (A) Representative flow cytometric plots of Spike-specific CD4+ CD154+ IFN- $\gamma^{+}$T cells in 1 naive (upper row) and 1 COVID-19-recovered (lower row) subject before vaccination and 7, 14, 21, and 28 days after the first injection. Kinetic analysis of frequencies of CD154+ cells producing at least 1 cytokine among IL-2, IFN- $\gamma$, and TNF- $\alpha$ (B), CD154 IFN- $\gamma^{+}$ (C), CD154+ IL-2+ (D), and CD154+ TNF- $\alpha^{+}$(E) Spike-specific T cells in naive (red lines) and COVID-19-recovered (blue lines) subjects before and after vaccination. Data are mean \pm SEM from 11 naive and 11 COVID-19-recovered subjects, subtracted from background unstimulated negative control. Blue and red asterisks refer to paired statistics within each study group compared with the previous time point in the kinetic. Black asterisks at day 28 represent paired statistic compared with prevaccine point. ${ }^{*} P<0.05$; ${ }^{* *} P<0.01$ calculated with Wilcoxon's signed rank test. (F) Characterization of SARS-CoV-2 Spikespecific CD4+ $T$ cell polyfunctionality in naive and COVID-19-recovered subjects at day 28 following the first vaccine injection. Results are expressed as mean percentages from 11 naive and 11 COVID-19-recovered subjects. (C) Characterization of TIGIT and PD1 expression by SARS-CoV-2 Spike-specific CD4 ${ }^{+}$ T cells in 7 naive and 8 COVID-19-recovered subjects. Results are expressed as mean percentages.

response, the frequency of Spike-specific $\mathrm{CD} 4^{+} \mathrm{T}$ cells showed a reduction in COVID-19-recovered subjects from day 7 to day 14 reaching statistical significance only for $\mathrm{CD} 154^{+} \mathrm{IFN}-\gamma^{+}$cells, and did not recover even after the second administration of the vaccine (Figure 3, B-E). On the contrary, as observed for B cells, T cells also showed a significant decrease following the second injection. Naive subjects instead showed a significant increase in the frequency of Spike-specific $\mathrm{CD} 4^{+} \mathrm{T}$ cells after the second dose, reaching the frequencies observed in COVID-19-recovered subjects (Figure 3, B-E). We then focused on the polyfunctional capability of Spike-specific CD $4^{+} \mathrm{T}$ cells in naive and in COVID-19recovered subjects at the end of the vaccination schedule (day 28 after the first injection). As shown in Figure 3F, we observed similar frequencies of Spike-specific $\mathrm{CD}^{+} \mathrm{T}$ cells producing 2 or 3 cytokines in combination. In agreement with these findings, we observed that Spike-specific $\mathrm{CD} 4^{+} \mathrm{T}$ cells displayed a similar expression pattern of 2 immune checkpoint molecules, $\mathrm{T}$ cell immunoreceptor with Ig and ITIM domains (TIGIT) and programmed cell death protein 1 (PD1) (Figure 3G). Altogether, these data demonstrate that Spike-specific $\mathrm{CD} 4^{+} \mathrm{T}$ cells, at least those identified based on CD154 and cytokine expression, have a similar functional potential at the end of the vaccination schedule both in naive and COVID-19-recovered subjects, independent of prior exposure to SARS-CoV-2. This is the first study, to our knowledge, evaluating the early kinetics of cellular immune response following 2 doses of BNT162b2 mRNA vaccine in naive and COVID-19recovered subjects. During the 3 weeks following the first vaccine injection antibodies, $\mathrm{B}$ and $\mathrm{T}$ cells specific for SARS-CoV-2 Spike protein progressively increased in naive subjects. In addition to what has been already demonstrated for antibodies and $\mathrm{T}$ cells in preclinical studies (11), B cell frequency also increases in the circulation after the administration of the second dose. Neutralizing antibody titers remain low, close to cut-off values, until day 21, but maximally increase after the second injection, confirming that the second dose is mandatory in naive individuals (12). Regarding COVID-19-recovered subjects, our findings suggest that there is a rapid reactivation of both humoral and cellular immunological memory to SARS-CoV-2 Spike protein. Indeed, 7 days after the first injection we observed maximal increase in circulating Spike-specific antibodies and $\mathrm{B}$ and $\mathrm{T}$ cells. Interestingly, we observed a decrease in the frequency of Spike-specific CD $4^{+} \mathrm{T}$ cells in COVID-19-recovered subjects 14 days after the administration of the first vaccine dose. We hypothesize that this reduction could be essentially linked to 2 phenomena: (a) sequestration of specific $\mathrm{CD} 4^{+} \mathrm{T}$ cells in the lymph node, or (b) suppression of specific $\mathrm{CD} 4^{+} \mathrm{T}$ cells through tolerogenic mechanisms. B cells do not display the same behavior, given that their frequency progressively increases until day 21 . On the contrary, the second injection appears to be ineffective, and it is rather associated to a contraction of both Spike-specific circulating $\mathrm{B}$ and $\mathrm{T}$ cells in the COVID-19-recovered group. Although these data depict the rapid kinetics of vaccine-induced immune response, they were obtained on a relatively small cohort. To further support these findings, we extended the serological evaluations on a larger cohort of vaccinated health care workers. Among 97 total subjects, 29 had a history of prior SARS-CoV-2 infection, dated 6 to 9 months before

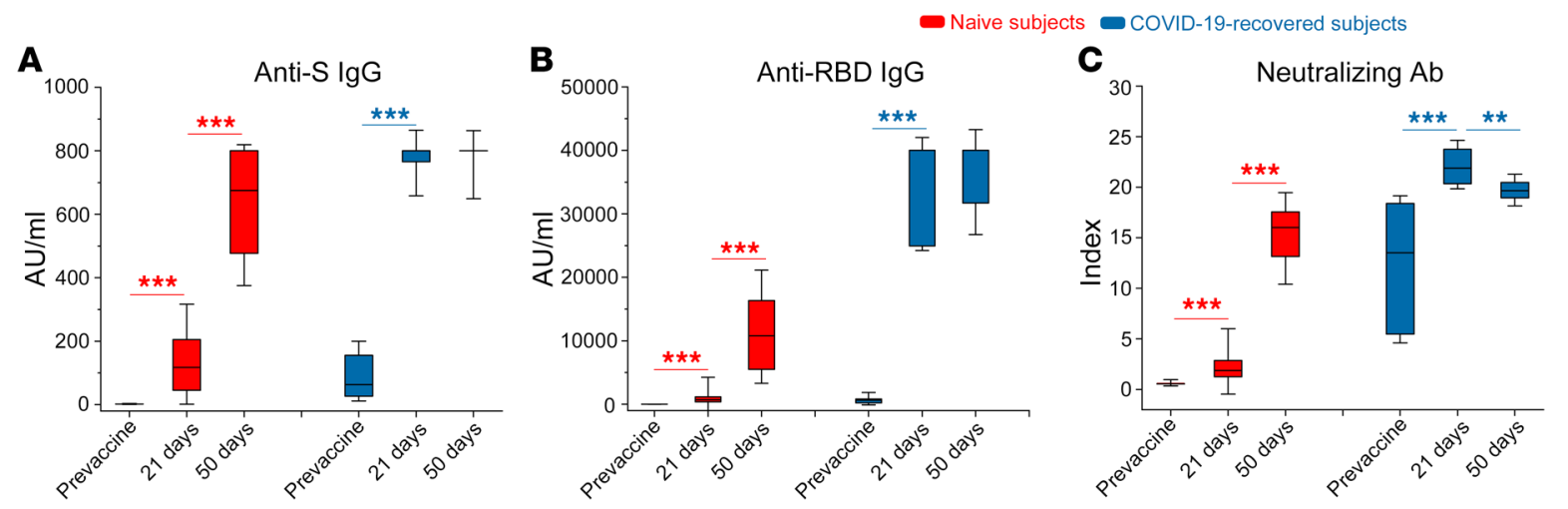

Figure 4. Characterization of vaccine-induced anti-Spike humoral response up to $\mathbf{5 0}$ days after vaccination. Evaluation of anti-S IgG (A), anti-RBD IgC (B), and S-neutralizing antibodies (C) in naive (red box) and COVID-19-recovered (blue box) subjects before and 21 and 50 days after first vaccine dose administration. Box-plots represent 25th to 75th percentiles. Black lines represent the median. Whiskers represent SE. Data were obtained from 68 naive and 29 COVID-19-recovered subjects. ${ }^{* *} P<0.01,{ }^{* *} P<0.001$ calculated with Wilcoxon's signed rank test. 
vaccination. Demographic and clinical data of this extended cohort are included in Supplemental Table 2. Subjects were tested before BNT162b2 mRNA vaccination, at day 21 (before the second injection) and at day 50. As shown in Figure 4, anti-Spike IgG, antiRBD IgG, and neutralizing Ab in naive individuals increased at day 21 and grew again at day 50. Before vaccination, COVID-19recovered subjects showed higher anti-Spike $\operatorname{IgG}(P<0.001$ with Mann-Whitney $U$ test), anti-RBD IgG $(P<0.001$ with Mann-Whitney $U$ test), and neutralizing $\mathrm{Ab}(P<0.001$ with Mann-Whitney $U$ test) than naive individuals. Anti-Spike and anti-RBD IgG levels maximized at day 21 and remained stable after the second injection until day 50 (Figure 4, A and B). Interestingly, neutralizing Ab peaked at day 21, but then significantly declined at day 50 (Figure 4C). Collectively, these data confirm what emerged from the study of vaccine-induced immunity in the smaller cohort. Our observations are in agreement with those recently published (13, 14) and those presented in an accompanying article in the JCI by Maria Rescigno's group (15), confirming that in COVID-19-recovered subjects, 1 dose of BNT162b2 is sufficient to maximize Spike-specific antibody titers. This information has practical fundamental implications. In most countries, individuals who recovered from SARS-CoV-2 infection are not excluded from COVID-19 vaccination, and they commonly follow a 2-dose vaccination schedule $(16,17)$. However, differently from naive individuals, our data suggest that 1 single injection may be sufficient to protect COVID-19-recovered subjects, in accordance with emerging data from other groups $(18,19)$. This is further supported by the results of clinical trials showing that the immunization levels induced by vaccination in naive subjects have $95 \%$ efficacy (6). We also cannot exclude that the second injection might even be detrimental in this context, possibly leading to a functional exhaustion of Spike-specific lymphocytes (20). Indeed, we observed a decrease in the frequency of both B and T cells at day 28 ( 1 week after second dose), but also a decline in the titer of neutralizing $\mathrm{Ab}$ at day 50. This is an intriguing hypothesis, but further studies are needed to fully prove it. Our data demonstrate that circulating Spikespecific $\mathrm{CD} 4^{+} \mathrm{T}$ cells have a similar polyfunctional capability and an immune checkpoint expression pattern in naive versus COVID-19-recovered subjects. However, we cannot exclude the presence of exhausted Spike-specific $\mathrm{CD} 4^{+} \mathrm{T}$ cells that cannot be reactivated ex vivo. It should be noted that our COVID-19-recovered cohort includes subjects with a history of symptomatic COVID-19 infection. It is known that the strength of the immune response to SARS-CoV-2 directly correlates to disease severity both in the acute phase $(21,22)$ and in the memory phase $(23)$ and that memory immune response to SARS-CoV-2 is heterogeneous in the months after recovery (24). For this reason, additional studies are required to understand whether a single mRNA vaccine administration may be sufficient also in people with a history of asymptomatic SARS-CoV-2 infection or in those presenting low levels of both humoral and cell mediated antigen-specific immune response. Additional information is also urgently needed on other approved COVID-19 vaccines. It should also be noted that our data were obtained on a cohort of subjects who experienced SARSCoV-2 infection 6 to 9 months before vaccination. Therefore, we do not know if a single vaccine injection might be equally effective in people who recovered from infection longer than 9 months before vaccination. Additional time is clearly needed to answer this question, given the recent outbreak of COVID-19.

Although approved by international and national medicines agencies, the distribution and administration of vaccines are still limited to a minority of the global population. Saving 1 vaccine dose for each person who recovered from COVID-19 would substantially increase the number of doses available for naive individuals, thus shortening the time to reach herd immunity.

\section{Methods}

Patients. Demographic and clinical information about the enrolled subjects is available in Supplemental Methods.

Flow cytometry. Full protocols for the identification of Spikespecific $\mathrm{T} \mathrm{CD} 4^{+}$and B cells are available in Supplemental Methods.

Detection of SARS-CoV-2-specific Ig. SARS-CoV-2-specific Ig and neutralizing $\mathrm{Ab}$ were quantitated following manufacturer's instructions, as detailed in Supplemental Methods.

Study approval. The procedures followed in the study were approved by the Careggi University Hospital Ethical Committee. Written informed consent was obtained from recruited patients.

\section{Author contributions}

AM, NDL, F Liotta, GMR, LC, AB, and FA designed the study. NDL, EM, MS, LZ, and F Lagi collected peripheral blood samples and collected informed consent. AM, LM, LS, AV, MC, GL, STK, MGC, and AR performed experiments. PP, CS, and LT provided advice. AM and LM analyzed data. AM, LS, and FA wrote the manuscript. All authors revised the manuscript and gave final approval.

\section{Acknowledgments}

We thank all the subjects who participated in the study. This study was supported by funds to the Department of Experimental and Clinical Medicine, University of Florence (Project Excellence Departments 2018-2022); the University of Florence, project RICTD2122; the Italian Ministry of Health (COVID-2020-12371849); and the Tuscany Region (TagSARS CoV 2). The COVID-19 Research Group is detailed in Supplemental Acknowledgments.

Address correspondence to: Francesco Annunziato, Department of Experimental and Clinical Medicine, University of Florence, Viale Pieraccini, 6, 50134 Florence, Italy. Email: francesco. annunziato@unifi.it.
1. World Health Organization. WHO Coronavirus Disease (COVID-19) Dashboard. https://covid19. who.int/. Updated April 26, 2021. Accessed on April 5, 2021.

2. Koh WC, et al. What do we know about SARS$\mathrm{CoV}-2$ transmission? A systematic review and meta-analysis of the secondary attack rate and associated risk factors. PLoS One. 2020;15(10):e0240205.

3. Marks M, et al. Transmission of COVID-19 in 282 clusters in Catalonia, Spain: a cohort study. Lancet Infect Dis. 2021;21(5):629-636.
4. Blumenthal D, et al. Covid-19 - implications for the health care system. N Engl J Med. 2020;383(17):1698.

5. Rosenbaum L. The untold toll - the pandemic's effects on patients without Covid-19. N Engl J Med.2020;382(24):2368-2371. 
6. Polack FP, et al. Safety and efficacy of the BNT162b2 mRNA Covid-19 vaccine. $N$ EnglJ Med. 2020;383(27):2603-2615.

7. Baden LR, et al. Efficacy and safety of the mRNA-1273 SARS-CoV-2 vaccine. $N$ Engl J Med. 2021;384(5):403-416.

8. Voysey M, et al. Safety and efficacy of the ChAdOx1 nCoV-19 vaccine (AZD1222) against SARSCoV-2: an interim analysis of four randomised controlled trials in Brazil, South Africa, and the UK. Lancet. 2021;397(10269):99-111.

9. Kyriakidis NC, et al. SARS-CoV-2 vaccines strategies: a comprehensive review of phase 3 candidates. NPJ Vaccines. 2021;6(1):28.

10. Wise J. Covid-19: people who have had infection might only need one dose of mRNA vaccine. BMJ. 2021;372:n308.

11. Sahin U, et al. COVID-19 vaccine BNT162b1 elicits human antibody and TH1 T cell responses. Nature. 2020;586(7830):594-599.

12. Walsh EE, et al. Safety and immunogenicity of two RNA-based Covid-19 vaccine candidates.
N Engl JMed. 2020;383(25):2439-2450.

13. Krammer F, et al. Antibody responses in seropositive persons after a single dose of SARS-CoV-2 mRNA vaccine. $N$ Engl J Med. 2021;384(14):1372-1374.

14. Bradley T, et al. Antibody responses after a single dose of SARS-CoV-2 mRNA vaccine. $N$ Engl J Med. 2021;384(20):1959-1961.

15. Levi R, et al. One dose of SARS-CoV-2 vaccine exponentially increases antibodies in individuals who have recovered from symptomatic COVID19. J Clin Invest. 2021;131(12): 149154.

16. Amit S, et al. Early rate reductions of SARS-CoV-2 infection and COVID-19 in BNT162b2 vaccine recipients. Lancet. 2021;397(10277):875-877.

17. Dagan N, et al. BNT162b2 mRNA Covid-19 vaccine in a nationwide mass vaccination setting. NEngl JMed. 2021;384(15):1412-1423.

18. Manisty C, et al. Antibody response to first BNT162b2 dose in previously SARSCoV-2-infected individuals. Lancet. 2021;397(10279):1057-1058.
19. Prendecki M, et al. Effect of previous SARSCoV-2 infection on humoral and T-cell responses to single-dose BNT162b2 vaccine. Lancet. 2021;397(10280):1178-1181.

20. Wherry EJ, Kurachi M. Molecular and cellular insights into T cell exhaustion. Nat Rev Immunol. 2015;15(8):486-499.

21. Mazzoni A, et al. Cell-mediated and humoral adaptive immune responses to SARS-CoV-2 are lower in asymptomatic than symptomatic COVID-19 patients. Eur J Immunol. 2020;50(12):2013-2024.

22. Sekine $\mathrm{T}$, et al. Robust $\mathrm{T}$ cell immunity in convalescent individuals with asymptomatic or mild COVID-19. Cell. 2020;183(1):158-168.

23. Mazzoni A, et al. Heterogeneous magnitude of immunological memory to SARS-CoV-2 in recovered individuals. Clin Transl Immunol. 2021;10(5):e1281.

24. Dan JM, et al. Immunological memory to SARSCoV-2 assessed for up to 8 months after infection. Science. 2021;371(6529): eabf 4063. 